\title{
Dissociable Neural Modulation Underlying Lasting First Impressions, Changing Your Mind for the Better, and Changing It for the Worse
}

\author{
Jamil P. Bhanji and Jennifer S. Beer \\ Department of Psychology, University of Texas at Austin, Austin, Texas 78712-1043
}

Unattractive job candidates face a disadvantage when interviewing for a job. Employers' evaluations are colored by the candidate's physical attractiveness even when they take job interview performance into account. This example illustrates unexplored questions about the neural basis of social evaluation in humans. What neural regions support the lasting effects of initial impressions (even after getting to know someone)? How does the brain process information that changes our minds about someone? Job candidates' competence was evaluated from photographs and again after seeing snippets of job interviews. Left lateral orbitofrontal cortex modulation serves as a warning signal for initial reactions that ultimately undermine evaluations even when additional information is taken into account. The neural basis of changing one's mind about a candidate is not a simple matter of computing the amount of competence-affirming information in their job interview. Instead, seeing a candidate for the better is somewhat distinguishable at the neural level from seeing a candidate for the worse. Whereas amygdala modulation marks the extremity of evaluation change, favorable impression change additionally draws on parametric modulation of lateral prefrontal cortex and unfavorable impression change additionally draws on parametric modulation of medial prefrontal cortex, temporal cortex, and striatum. Investigating social evaluation as a dynamic process (rather than a one-time impression) paints a new picture of its neural basis and highlights the partially dissociable processes that contribute to changing your mind about someone for the better or the worse.

\section{Introduction}

Research has shown that while we are not enslaved to our first impressions of other people, we are not always completely free of them either. A central tenet of social evaluation research is that people form spontaneous impressions of others and that this process is imperfect. For example, people tend to initially form impressions of someone's ability or moral character using irrelevant information such as physical attractiveness (i.e., "the halo effect") (Dion et al., 1972; Eagly et al., 1991; Feingold, 1992; Jackson et al., 1995; Srivastava et al., 2010). Although subsequent information may counter initial impressions, it is not always enough to override those initial impressions. Managers and teachers often assume that unattractive candidates or students are less competent, intelligent, and socially skilled and these biases do not completely disappear even when new information points to equivocal skills across attractive and unattractive people (Eagly et al., 1991; Feingold, 1992; Jackson et al., 1995). Although social evaluations typically unfold through a combination of initial impressions and modifications related to subsequent infor-

\footnotetext{
Received Dec. 10, 2012; revised April 8, 2013; accepted April 22, 2013

Author contributions: J.P.B. and J.S.B. designed research; J.P.B. performed research; J.P.B. analyzed data; J.P.B. and J.S.B. wrote the paper.

This work was funded by National Science Foundation BCS 0746017 to J.S.B.

The authors declare no competing financial interests.

Correspondence should be addressed to Jamil Palacios Bhanji, Department of Psychology, Rutgers UniversityNewark, 101 Warren Street, Newark, NJ 07201. E-mail: bhanj@utexas.edu.

DOI:10.1523/JNEUROSCI.5634-12.2013

Copyright $\odot 2013$ the authors $\quad 0270-6474 / 13 / 339337-08 \$ 15.00 / 0$
}

mation, research on neural substrates of social evaluation has largely focused on one-time, static impressions. Recently, a few studies have made strides in understanding the dynamics of social evaluation by manipulating the inconsistency of person information (Croft et al., 2010; Harris and Fiske, 2010; Baron et al., 2011; Cloutier et al., 2011; Ma et al., 2012; Mende-Siedlecki et al., 2013). Research has not yet addressed neural responses underlying (1) the undermining effects of initial impressions or (2) the incorporation of new information, which demonstrably changes impressions for the better or for the worse. The current study bridges this gap by using dynamic social stimuli to investigate the neural activity associated with initial impressions and their modification.

The current study capitalizes on the well established finding that evaluations of competence arise from a combination of an initial evaluation of physical attractiveness and subsequent information about ability (Eagly et al., 1991; Jackson et al., 1995). Participants' evaluations of unattractive and attractive job candidates were dynamically tracked as they viewed first a candidate's picture and then the candidate's statements in a job interview. Although job interviews portrayed different levels of competence, they were equivalent for unattractive and attractive job candidates across the experiment. The study addresses the neural responses that support the "damage done" by initial impressions of physical attractiveness, that is, the influence that physical attractiveness has even after information that is pertinent to a person's competence becomes available. Furthermore, the study addresses the neural responses that support the modification of 
initial impressions for the better or worse. A number of neural regions are associated with processing inconsistent information about a person (medial prefrontal cortex (MPFC), striatum, and lateral temporal cortex; Croft et al., 2010; Harris and Fiske, 2010; Baron et al., 2011; Cloutier et al., 2011; Ma et al., 2012, Mende-Siedlecki et al., 2013). Yet it remains unknown whether their role is affected by (1) the degree to which that information actually changes an evaluation of someone or (2) the direction of evaluation change. What neural regions support the undermining effects of initial evaluations even when new information is taken into account? What neural regions process information that changes someone's mind about a candidate for the better or worse after an initial evaluation has occurred?

\section{Materials and Methods}

Twenty-five female participants underwent functional magnetic resonance imaging (fMRI) while judging pictures and videos of putative male job candidates (ages $18-21$ years, mean age $=18.6, \mathrm{SD}=0.82$ ). Only female participants were enrolled in the study to eliminate possible confounds between different gender pairings of judges and targets (Eagly et al., 1991; Feingold, 1992). Participants were paid $\$ 30(n=10)$ or received course credit $(n=15)$. All participants provided informed consent and the study was approved by the institutional review board of the University of Texas at Austin.

The experiment included photos and job interview snippets (video clips with audio) of job candidates (20 Unattractive, 20 Average Attractiveness) who were supposedly interviewing for an open position at a large corporation. The stimuli were developed by the first author for the purposes of this experiment. Standardized photographs and video clips were developed from a pool of actors who posed as job candidates and performed a scripted job interview. Fifty actors were recruited through a website for acting and voice-over job postings. Photographs were standardized for attractiveness. The 40 job candidates shown in the study were selected based on ratings of physical attractiveness from a separate sample of 40 female judges drawn from the same subject pool used in the fMRI study (University of Texas students). Judges rated the physical attractiveness of each candidate on a scale from 1 (not at all attractive) to 5 (very attractive). The goal of the study was to understand the disadvantage of low physical attractiveness on impressions. Therefore, the study involved 20 candidates assigned to the Unattractive condition ( mean $=$ $1.54, \mathrm{SD}=0.33$ ) and 20 candidates assigned to the Average Attractiveness condition $($ mean $=2.91, \mathrm{SD}=0.54$; attractiveness was significantly different between the two groups $\left(t_{(39)}=22.64, p<0.001\right)$. Job interview snippets consisted of 120 scripted job interview statements (60 high competence: "I appreciate constructive criticism from coworkers and superiors"; 60 low competence: "I need to take a cigarette break every hour or I can get kind of cranky"). Four judges rated each statement on a scale from 1 (low competence) to 5 (high competence) as well as categorized the statements as indicating "high" versus "low" competence. Low competence statements were judged as indicating lower competence $($ mean $=1.02, \mathrm{SD}=0.07)$ than high competence statements (mean $=$ $\left.4.67, \mathrm{SD}=0.39, t_{(3)}=65.38, p<0.001\right)$. Reliability of the judges' ratings was high (Cronbach's $\alpha=0.996$ ) and the four judges had $100 \%$ agreement when categorically judging whether the statement was low or high competence. Unattractive and Average Attractiveness candidates made the same statements, which permitted counterbalanced job interview information across attractiveness conditions.

Participants acted as a hiring manager and evaluated each candidate's competence on a scale (1: low to 5: high). Each trial consisted of an Initial Evaluation and a Final Evaluation (Fig. 1). In the Initial Evaluation, participants viewed a candidate's photograph $(2.5 \mathrm{~s})$ and then evaluated that candidate's competence (2.5 s). Participants then saw a cue to clear their minds (fixation screen, $7.5 \mathrm{~s}$ ). In the Final Evaluation, participants viewed three job interview snippets of the same candidate $(16 \mathrm{~s})$ and then made a second evaluation of that candidate's competence $(2.5 \mathrm{~s})$. The trial ended by cuing participants to clear their minds (fixation screen, $16.5 \mathrm{~s}$ ). Four levels of experimentally manipulated candidate competence were achieved by varying the ratio of high and low competence statements within a block. That is, participants saw candidates make three low competence statements (Low objective competence), two low and one high competence statements (Mostly Low objective competence), one low and two high competence statements (Mostly High objective competence), or three high competence statements (High objective competence). Unattractive and Average Attractive candidates were portrayed with equal levels of competence through counterbalanced assignment of ratios of job interview information. Furthermore, the pairing of particular job interview information was randomly assigned to candidates and randomly ordered within blocks.

\section{Competence evaluation analysis}

Behavioral data analyses examined (1) the influence of job interview information on changes in competence evaluations, (2) the initial influence of physical attractiveness on competence evaluations, and (3) the continuing influence of attractiveness on competence evaluations after seeing the job interviews. No significant differences in competence ratings in any of the conditions were found between participants who were compensated with money compared with those compensated with course credit (all $t_{(23)}<2, p>0.10$ ). Therefore, data from all 25 participants were analyzed together. The factors affecting changes in evaluation (Final Evaluation minus Initial Evaluation) were tested with a 4 (Job Interview Information: $0,1,2$, or 3 high competence statements $) \times 2$ (Attractiveness: Unattractive or Average Attractiveness) ANCOVA. This analysis permitted a test of whether job interview information or attractiveness influenced the degree to which participants changed their evaluation (main effect of Job Interview Information or Attractiveness, respectively), and whether the influence of job interview information differed for Unattractive versus Average Attractiveness candidates (interaction). Increasing levels of competence in the Job Interview Information were expected to increase evaluation change. Therefore, a linear contrast was used to test for effects of Job Interview Information (main effect and interaction with Attractiveness). To understand magnitude of evaluation change independently of any differences in absolute level of initial competence ratings, the ANCOVA controlled for Initial Evaluations of competence. Paired $t$ tests were conducted to investigate effects of Attractiveness on Initial and Final Evaluations (competence ratings and response times). Additionally, an Attractiveness manipulation check was conducted to verify differences in physical attractiveness ratings of the Unattractive and Average Attractiveness candidates, which participants made after exiting the scanner.

\section{fMRI acquisition and preprocessing}

Images were collected on a 3.0 T GE Signa EXCITE scanner. Blood oxygen level-dependent functional images were acquired with an echoplanar imaging sequence $(\mathrm{TR}=2500 \mathrm{~ms}, \mathrm{TE}=30 \mathrm{~ms}, \mathrm{FOV}=220 \mathrm{~mm}$, $64 \times 64$ matrix, 32 axial slices $3 \mathrm{~mm}$ thick, $0.5 \mathrm{~mm}$ gap, voxel size $3.44 \times$ $3.44 \times 3.5 \mathrm{~mm})$. Functional volume acquisitions were time locked to the onset of each trial. A high resolution T1-weighted structural image and a 
T2-weighted structural image coplanar with the functional scan were collected. Functional images were collected in four consecutive runs (10 trials each run). Hardware problems resulted in the loss of one run for one participant, analyses for this participant were conducted on the available data (30 trials of a total 40 trials of the task). Neuroimaging data were preprocessed and analyzed with FMRIB's Software Library version 4.1 (FSL; http://www.fmrib.ox.ac.uk/fsl/; Smith et al., 2004). Functional images were corrected for head motion, then corrected for slice-timing skew, then high-pass filtered to remove low-frequency noise (cutoff period $47.5 \mathrm{~s}$ equal to the length of a full trial). Data were resampled to 2 $\mathrm{mm}$ cubic resolution and spatially smoothed with a $5 \mathrm{~mm}$ full-width at half-maximum isotropic Gaussian kernel. Functional images were spatially normalized into the Montreal Neurological Institute (MNI) standard, using the T2-weighted structural image for initial registration and the high resolution T1-weighted structural image for registration to the MNI T1 template.

\section{fMRI analysis}

Functional images were analyzed to identify neural activity associated with the lingering effect of attractiveness on final competence evaluations (Goal 1), parametrically modulated by changes in participants' subjective evaluations for each candidate (Goal 2), and parametrically modulated by the objective competence level (Goal 3). As described below, achieving these three goals required two general linear models (GLMs).

GLM specification (goals 1 and 2). The GLM consisted of eight regressors of interest and six regressors of noninterest. Two regressors of interest modeled the Initial Evaluation period, that is, the candidate photographs and Initial Evaluation rating (photograph onset to rating offset, $5 \mathrm{~s}$ duration, one regressor for Attractive candidates, one regressor for Unattractive candidates). The third and fourth regressors of interest modeled the Final Evaluation period, that is, the presentation of job interview snippets and Final Evaluation ratings (onset of job interview snippets to rating offset, $18.5 \mathrm{~s}$ duration, separate regressors for the Unattractive and Average Attractiveness conditions). The fifth and sixth regressors of interest tested for neural responses during the Final Evaluation period that were parametrically modulated by changes in subjective evaluations (separate regressors for the Unattractive and Average Attractiveness conditions). Specifically, these Linear Modulation regressors were time locked to the Final Evaluation period regressor and weighted by the subjective evaluation change on each trial (Final Evaluation minus Initial Evaluation competence rating for each candidate). The seventh and eight Quadratic Modulation regressors of interest reflected the square of each Linear Modulation regressor and tested for neural responses during the Final Evaluation that showed a curvilinear relation to subjective evaluation change. Six regressors of noninterest modeled participant head movement during the scan. The regressors for Final Evaluation, Linear Modulation, and Quadratic Modulation were serially orthogonalized (Büchel et al., 1998). These four regressors and the two Initial Evaluation period regressors were convolved with a canonical double-gamma response function in FSL's FEAT first-level analysis package.

Group-level analysis. Least-squares estimates were used to calculate contrast maps for the four functional scans for each participant and then averaged across scans for each participant. Contrasts estimated the effect of candidate attractiveness on neural activity (Unattractive vs Average Attractiveness and vice versa), linear modulation by subjective evaluation change (increasing or decreasing), and quadratic modulation by evaluation change (increasing with extremity of change or decreasing with extremity of change). Group level random effects analysis used the FMRIB's Local Analysis of Mixed Effects (FLAME) approach in FSL. A cluster correction threshold was applied to group level $z$-statistic maps (cluster threshold of $z>2.3$, corrected cluster significance threshold of $p<0.05)$.

Analysis (goal 1): identify neural activity associated with the lingering effect of attractiveness on final competence evaluations. One set of tests investigated how neural responses to candidate attractiveness related to behavioral indices of competence evaluations. Percentage signal change was extracted from significant clusters found in the relevant group contrast (i.e., Unattractive $>$ Average Attractiveness which was cluster cor- rected at $p<0.05$ for the whole brain; Kriegeskorte et al., 2009) and tested for significant correlation with attractiveness effects on reaction times and competence ratings at the two evaluation time points (i.e., Unattractive Initial Evaluation ratings minus Average Attractiveness Initial Evaluation ratings, Unattractive Final Evaluation ratings minus Average Attractiveness Final Evaluation ratings, etc.). Regions were selected from the whole brain contrast rather than on an a priori basis because previous literature has not examined how neural activity is related to initial impressions that continue to influence evaluations even when new information does not support the initial impressions. Correlation tests were corrected for the number of activation clusters identified in the main contrast (only one activation cluster in left lateral orbitofrontal cortex (LOFC) was identified in the main contrast).

Analysis (goal 2): identify neural activity parametrically modulated by changes in participants' subjective evaluations for each candidate. Activation clusters significantly associated with the linear parametric regressors were interpreted on the basis of their relation to favorable or unfavorable changes in evaluations of competence. Consistent with the behavioral data, which found that competence information was used to the same extent for Unattractive and Average Attractiveness candidates, Attractiveness did not influence the neural regions modulated by changes in evaluations in competence. Therefore, group-level contrast results are presented collapsed across the Unattractive and Average Attractiveness conditions. The neural association to seeing someone for the better was interpreted as clusters whose activation linearly increased to the degree that participants changed their evaluations of competence in a favorable direction. The neural association to seeing someone for the worse was interpreted as clusters whose activation linearly increased to the degree that participants changed their evaluations of competence in an unfavorable direction. An additional region of interest analysis was conducted to specifically test for evidence of a quadratic effect of subjective evaluation change in the amygdala. As in previous research examining quadratic effects of initial social evaluation in the amygdala (Cunningham et al., 2008; Said et al., 2009), a small volume correction for familywise error $(p<0.05)$ was applied in bilateral amygdala (Harvard-Oxford probabilistic atlas: 50\% probability threshold; Desikan et al., 2006). For visualization, percentage signal change values were extracted from significantly activated clusters in the linear or quadratic modulation analyses and plotted according to degree of subjective evaluation change (see Figs. 3-5). Furthermore, these findings hold even if the analyses take absolute level of initial competence evaluations into account. If the GLM is estimated including an additional regressor of noninterest that was time locked to the Final Evaluation regressor and weighted by initial competence evaluations on each trial, the results remain the same.

GLM specification (goal 3). A final goal was to understand convergence between neural modulation related to subjective changes in competence evaluation and modulation related to experimentally manipulated levels of competence. Therefore, a separate GLM examined neural activity parametrically modulated by the experimentally manipulated levels of job competence. The four regressors of interest included two regressors modeling the Final Evaluation period (one for Unattractive, one for Average Attractiveness) and two regressors modeling the linear parametric modulation related to the experimentally manipulated levels of competence (one for Unattractive, one for Average Attractiveness). Specifically, the linear parametric modulation regressors were weighted by the level of experimentally manipulated competence $($ Low $=1$, Mostly Low $=2$, Mostly High $=3$, High $=4$, time locked and orthogonalized to the Final Evaluation period regressors). Two regressors of noninterest modeled the other portions of the experiment (i.e., the Initial Evaluation for Unattractive, Average Attractiveness candidates). Six additional regressors of noninterest modeled head movement. Regressors (except those associated with head movement) were convolved with a canonical doublegamma response function.

Group-level analysis and thresholding proceeded as described for the first GLM. As in the analysis for Goal 2, group-level contrast results are presented collapsed across the Unattractive and Average Attractiveness conditions. Two group maps were of interest: neural activity that increased in relation to greater competence information and neural activity that increased in relation to lower competence information. For visual- 
Table 1. Mean (and SD) initial and final competence evaluations and response times for Unattractive and Average Attractiveness candidates

\begin{tabular}{|c|c|c|c|c|c|c|}
\hline & \multirow[b]{2}{*}{ Initial evaluation } & \multicolumn{5}{|c|}{ Final evaluation (as a function of job interview competence level) } \\
\hline & & All competence levels & Low & Mostly low & Mostly high & High \\
\hline \multicolumn{7}{|l|}{ Unattractive } \\
\hline Competence & $3.08(0.52)$ & $2.66(0.26)$ & $1.14(0.25)$ & $1.77(0.34)$ & $3.14(0.53)$ & $4.54(0.45)$ \\
\hline \multicolumn{7}{|c|}{ Average Attractiveness } \\
\hline Competence & $3.76(0.56)$ & $2.75(0.24)$ & $1.24(0.33)$ & $1.91(0.50)$ & $3.15(0.55)$ & $4.71(0.34)$ \\
\hline RT & 752 ms (400) & 866 ms (199) & $711 \mathrm{~ms}(232)$ & $986 \mathrm{~ms}(289)$ & 977 ms (348) & $787 \mathrm{~ms}(230)$ \\
\hline
\end{tabular}

RT, response times.

ization (Figs. 3-5), percentage signal change values were extracted from significant activation clusters and plotted for each level of experimentally manipulated competence. Finally, a conjunction analysis identified neural regions that process new information (manipulated level of competence) that actually changes someone's mind (subjective change in competence evaluation) about a candidate. Specifically, we tested the conjunction between the linear modulation associated with manipulated competence (second GLM) and the linear modulation associated with subjective evaluation change (first GLM) (Nichols et al., 2005).

\section{Results}

Competency-related information changes subsequent evaluations of candidates, but skin-deep information remains influential

The job interview information as well as persistent effects of attractiveness contributed to final evaluations. The extent to which participants used the job interview information to change their evaluation was tested with a 4 (job interview information: $0,1,2$, or 3 high competence statements made by the candidate) $\times 2$ (attractiveness: Unattractive or Average Attractiveness) ANCOVA. Change in evaluation (i.e., discrepancy between Final Evaluation and Initial Evaluation) was significantly shaped by a linear effect of job interview information $\left(F_{(3,20)}=7.89, p=0.010\right)$. Participants significantly changed their evaluations of candidate's competence in a manner that was commensurate with the degree of competenceconsistent information in the job interview (Table 1). For example, the more job interview information affirmed competence, the more evaluations of competence changed for the better. There were no significant relations found for the main effect of attractiveness $\left(F_{(1,22)}=1.33, p=0.261\right)$ or the interaction term $\left(F_{(1,20)}=2.03, p=0.168\right)$. That is, participants did not significantly differ in how much they used job interview information to change their impressions of unattractive compared with average attractiveness candidates. The manipulation check showed that the attractiveness manipulation was effective: Unattractive candidates were rated as less physically attractive $($ mean $=1.61, \mathrm{SD}=0.39)$ than Average Attractiveness candidates ( mean $=2.81, \mathrm{SD}=0.51 ; t_{(24)}=$ $17.37, p<0.001$ ) in post scan ratings.

Although participants used the new information to change their impressions of both unattractive and average attractiveness candidates, skin-deep information still affected both initial and final evaluations of competence. Unattractive candidates were evaluated as less competent than averagely attractive candidates in the Initial Evaluation period $\left(t_{(24)}=-6.56, p<0.001\right)$. Most importantly, unattractiveness continued to affect evaluations of competence even after participants saw the job interviews $\left(t_{(24)}=\right.$ $-2.47, p=0.021)$. Even though job interview information was counterbalanced such that unattractive and averagely attractive candidates communicated equivalent levels of competence, unattractive candidates were still evaluated as less competent in the final evaluation. Attractiveness only showed a main effect on reaction times for the Initial Evaluation. Unattractive candidates were evaluated more slowly in the Initial Evaluation period (Unattractive mean $=822 \mathrm{~ms}, \mathrm{SD}=401$; Average Attractiveness mean $=752 \mathrm{~ms}, \mathrm{SD}=400 ; t_{(24)}=2.37, p=0.026$ ).

\section{Left lateral orbitofrontal activity predicts the "damage done" by physical attractiveness}

LOFC activity predicted the way in which attractiveness influenced final evaluations of competence even when they could be based on additional information from the job interviews. That is, the more that LOFC activation was modulated by photograph attractiveness, the more participants ultimately evaluated unattractive candidates as less competent despite their equivalent job interview performance. The Unattractive compared with Attractive photograph contrast found significant activation in the LOFC (Fig. 2; peak $z=$ 3.46, MNI $x, y, z=-42,20,-10$, Brodmann's area 47, 504 voxels, $p<0.05$, cluster corrected). This LOFC region was the only cluster identified in the contrast of Unattractive versus Average Attractiveness photographs. Activity in this LOFC cluster correlated with individual differences in how much Unattractiveness influenced (1) response times at Initial Evaluation $(r=0.60, p=0.002$, corrected for the number of clusters from the main contrast) and (2) competence ratings at Final Evaluation $(r=0.43, p=0.030$; corrected for the number of clusters from the main contrast). That is, the more participants showed LOFC modulation in response to Unattractiveness in the Initial Evaluation, the more slowly they evaluated Unattractive candidates. Furthermore, the same LOFC modulation predicted lower competence evaluations of Unattractive candidates in the Final Evaluation.

\section{Seeing someone for the better: positive parametric relation to} right lateral prefrontal cortex activity

The extent to which job interview information increased evaluations of competence was associated with right lateral prefrontal cortex (LPFC) activation. A region within LPFC responded to parametrically increasing levels of (1) objective competence information and (2) subjective favorable evaluation change (Fig. 3, Table 2). That is, LPFC modulation tracked the extent to which job interviews conveyed increasing levels of competence (Fig. $3 B$ ) and the extent to which candidates were ultimately evaluated as better than initially thought (evaluations decreased from Initial Evaluation to the Final Evaluation for a particular candidate: Fig. 3C).

Seeing someone for the worse: negative parametric relation to MPFC, striatum, and lateral temporal cortex

The extent to which job interview information decreased evaluations of competence was associated with MPFC, striatum, and lateral temporal cortex (superior temporal sulcus and middle temporal gyrus) activation. That is, consistent regions of MPFC, striatum, and lateral temporal cortex responded to (1) parametrically decreasing levels of objective competence information and (2) parametrically decreasing subjective unfavorable evaluation change (Fig. 4, Table 2). That 


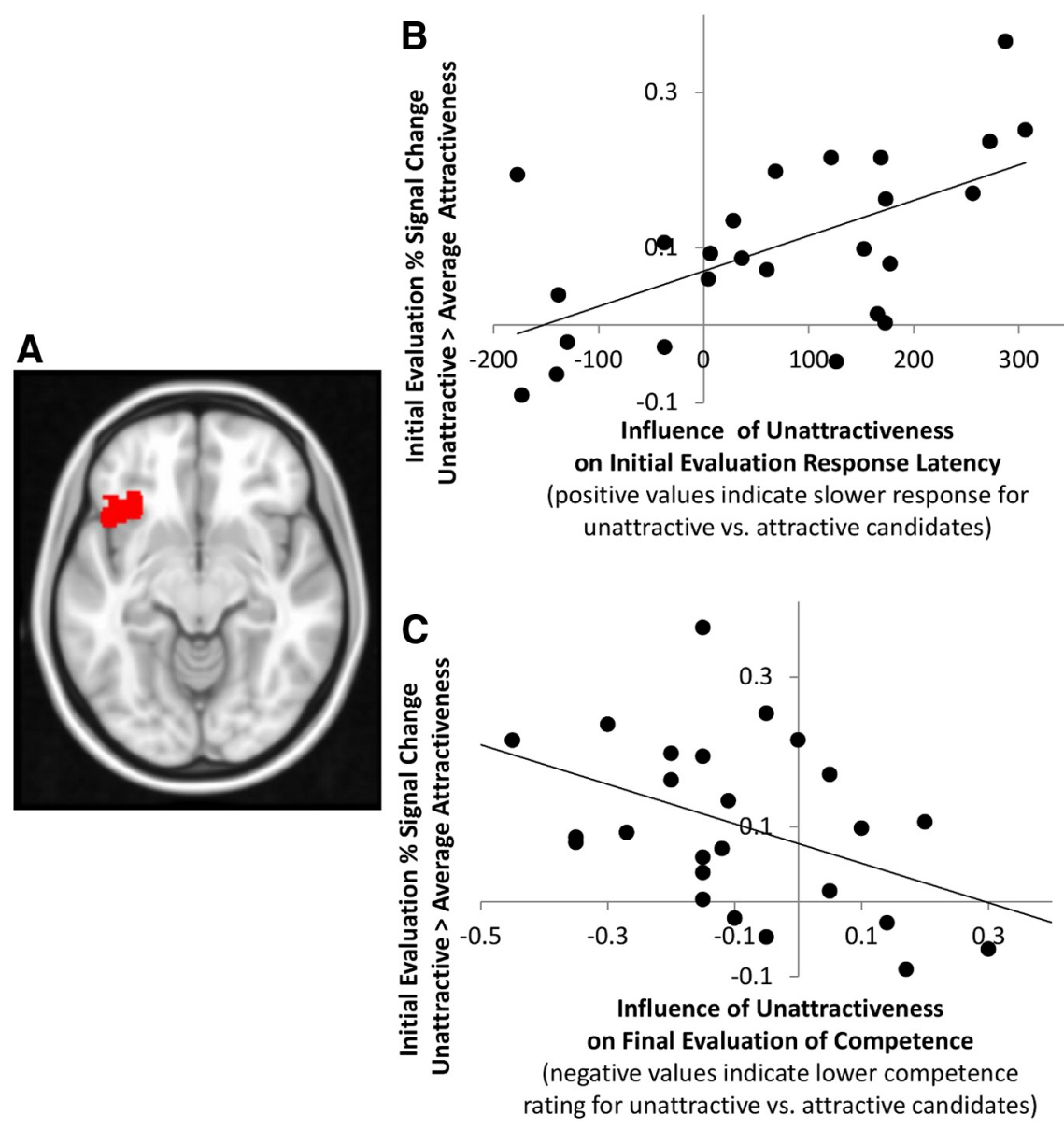

Figure 2. LOFC activity elicited by physically unattractive compared with average attractiveness photographs predicts the extent to which final evaluations are more unfavorable for unattractive candidates (despite equivalent levels of competence in the job interview as average attractiveness candidates). $\boldsymbol{A}$, Left LOFC region where activity was significantly greater for Unattractive compared with Average Attractiveness candidates. B, LOFC signal change for Unattractive compared with Average Attractiveness candidates (vertical axis) correlates with the additional time spent on Initial Evaluations of Unattractive compared with Average Attractiveness candidates (horizontal axis). C, LOFC signal change for Unattractive compared with Average Attractiveness candidates (vertical axis) correlates with the damage done by physical unattractiveness on Final Evaluations of competence (horizontal axis: competence ratings of Unattractive minus Average Attractiveness candidates).

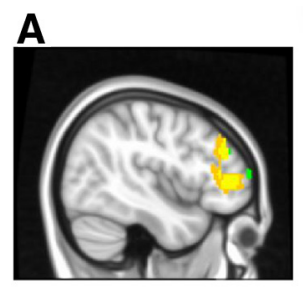

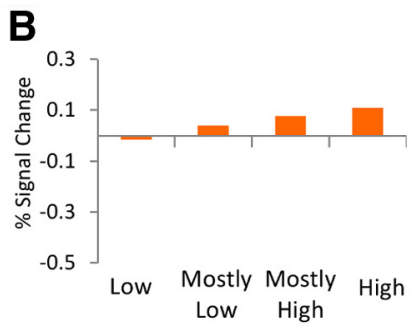

Objective Competence

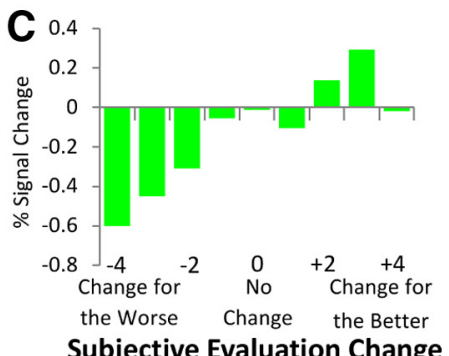

Subjective Evaluation Change

Figure 3. Illustration of parametric neural modulation and seeing a candidate for the better. $A$, Depicts voxels showing a parametric relation to experimentally manipulated competency-related information (orange), changes in subjective competence evaluations (green), or both effects (yellow). $\boldsymbol{B}$, Average percentage signal change is plotted in relation to experimentally manipulated levels of competence (averaged across orange and yellow voxels). C, Average percentage signal change is plotted in relation to how much participants had a favorable change of heart in their final evaluations of a candidate (averaged across green and yellow voxels). Positive values on the horizontal axis indicate that the final evaluation of the candidate's competence was higher than the initial evaluation.

is, MPFC, striatum, and lateral temporal cortex modulation tracked the extent to which job interviews conveyed decreasing levels of competence (Fig. $4 B, E, H, K$ ) and the extent to which candidates were ultimately evaluated as worse than initially thought (eval- uations of competence decreased from Initial Evaluation to the Final Evaluation for a particular candidate; Fig. $4 C, F, I, L)$.

\section{Seeing some for better or worse: quadratic parametric relation to amygdala activity}

Whereas some neural regions were associated with change in evaluation for the better or the worse, amygdala activation more broadly marked the extremity of evaluation change in any direction. The extent to which job interview information changed evaluations of competence showed a quadratic relation to bilateral amygdala activation (right peak at $28,-2,-16, z=2.88,32$ voxels; left peak at $-24,-2,-14, z=2.57,7$ voxels). The more participants recruited bilateral amygdala to process the job interview information, the more they changed their minds about the candidate (lowest activation for no change; Fig. 5).

\section{Discussion}

Even when we are open to seeing people in a new light, we cannot always completely shake off our initial reactions. In everyday life, physically unattractive people are subject to less favorable evaluations despite equivalent performance at work (Dion et al., 1972; Eagly et al., 1991; Feingold, 1992; Jackson et al., 1995; Srivastava et al., 2010). In the present study, initial LOFC response to unattractive job candidates predicted less favorable final evaluations of competence despite equivalent job interview performance. The extent to which people change their minds about a job candidate was reflected in quadratic modulation of amygdala, which was complemented by linear parametric modulation of (1) LPFC activation when they changed their minds for the better or (2) MPFC, striatum, and lateral temporal cortex when they changed their minds for the worse. The current study provides a number of novel insights into social evaluation through its investigation of the neural substrates underlying the dynamics between initial reactions and the incorporation of new information into social perception.

\section{LOFC: the ironic effects of suppressing negative attitudes toward unattractive people?}

LOFC engagement provides a warning signal that, despite new information, initial reactions will color ultimate evaluations. In the present study, LOFC modulation in response to unattractive candidates marked the extent to which initial evaluations (1) were made more slowly and (2) undermined final evaluations despite knowing much more about the person. These findings demonstrate that focusing on one- 


\begin{tabular}{|c|c|c|}
\hline \multirow[b]{2}{*}{ Region, Brodmann area } & \multirow{2}{*}{$\begin{array}{l}\text { Relation to objective manipulation } \\
\text { of new information } \\
\text { Peak }(x, y, z), z \text {-statistic, \#voxels }\end{array}$} & \multirow{2}{*}{$\begin{array}{l}\begin{array}{l}\text { Relation to subjective evaluation } \\
\text { change }\end{array} \\
\text { Peak }(x, y, z), z \text {-statistic, \#voxels }\end{array}$} \\
\hline & & \\
\hline \multicolumn{3}{|c|}{$\begin{array}{l}\text { Favorable change (increased activity tracks the extent of objective positive information and the extent to which } \\
\text { subjective evaluations change for the better }\end{array}$} \\
\hline Right LPFC, 45 & $(44,40,4), 5.41,1277$ & $(38,60,14), 3.41,649$ \\
\hline Right inferior parietal, 40 & $(52,-48,44), 4.81,1813$ & $(60,-46,44), 3.41,544$ \\
\hline Right occipital, 17 & $(10,-84,12), 5.00,963$ & $(14,-80,-14), 4.51,977$ \\
\hline Left MPFC, 32 & $(-8,50,28), 6.23,5896$ & $(-8,50,26), 5.49,6189$ \\
\hline Left/right striatum & $(-10,10,4), 5.27,3331$ & $(-4,-8,4), 4.47,3167$ \\
\hline Right lateral temporal, 20 & $(50,2,-32), 6.31,1768$ & $(52,-2,-38), 5.35,2915$ \\
\hline Left lateral temporal, 20 & $(-54,-6,-30), 7.24,4559$ & $(-56,-2,-26), 5.91,5110$ \\
\hline
\end{tabular}

time evaluations can obscure the full role that neural regions play as social evaluation unfolds across initial reactions to later evaluations. Furthermore, the relation between LOFC activity and response slowing raises two possibilities for the precise role of LOFC in evaluating others. In the current study, LOFC activation may reflect negative attitudes that were initially masked by taking longer to report an initial impression. This possibility is consistent with previous research finding an association between LOFC activity and the magnitude of negative attitudes toward a social target (O’Doherty et al., 2003; Beer et al., 2008) and the extent to which those negative attitudes affect social interactions (Kim et al., 2012). For example, LOFC activity predicts appearance-based biases in economic interactions with others. Specifically, LOFC activity related to participants' tendency to reject ultimatum game offers from untrustworthylooking partners at a greater rate than offers from trustworthy-looking partners (Kim et al., 2012). Alternatively, LOFC activation may reflect the control efforts exerted when reporting an initial impression. Across numerous domains, LOFC activation is associated with inhibitory efforts (Casey et al., 1997; Elliot et al., 2000). Consequently, LOFC activation may predict the negativity of final evaluations when new information is not enough to counteract initial negative expectations or when initial control efforts are no longer sustained.

Neural distinctions between changing your mind about someone for the better, for the worse, and to the extreme The current study also paints a more complete picture of the neural basis of social evaluation by investigating what happens when people change their mind about someone. Although one neural region marked the extremity of evaluation
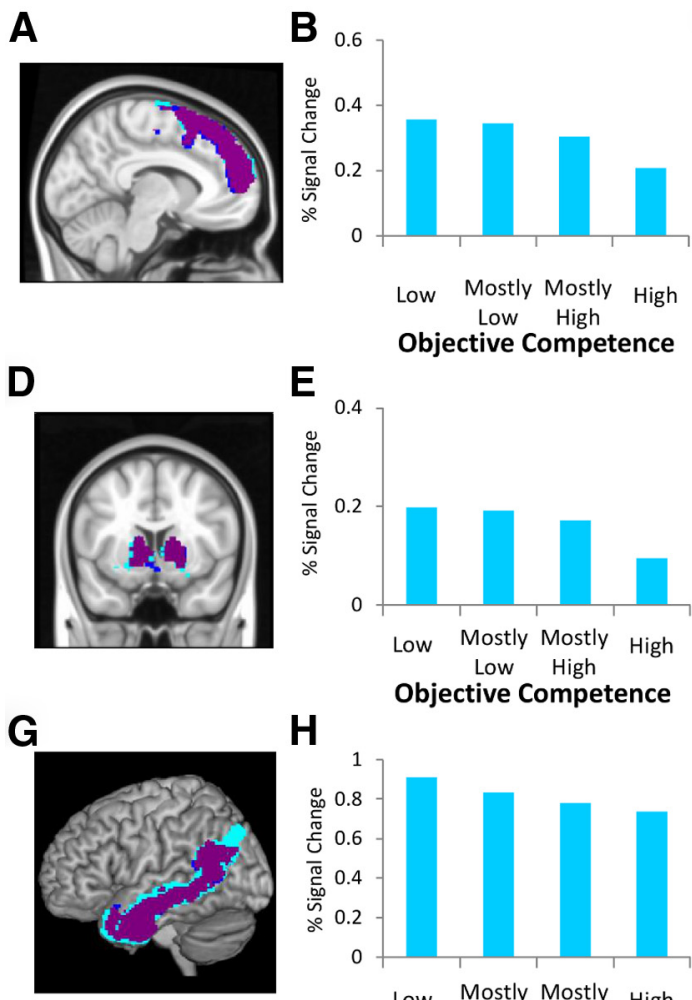

H

Objective Competence

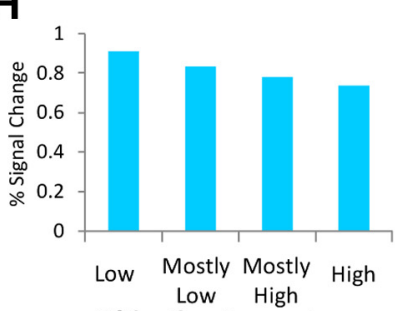

Objective Competence

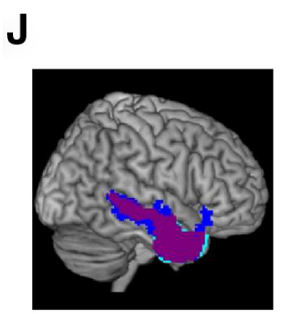

K
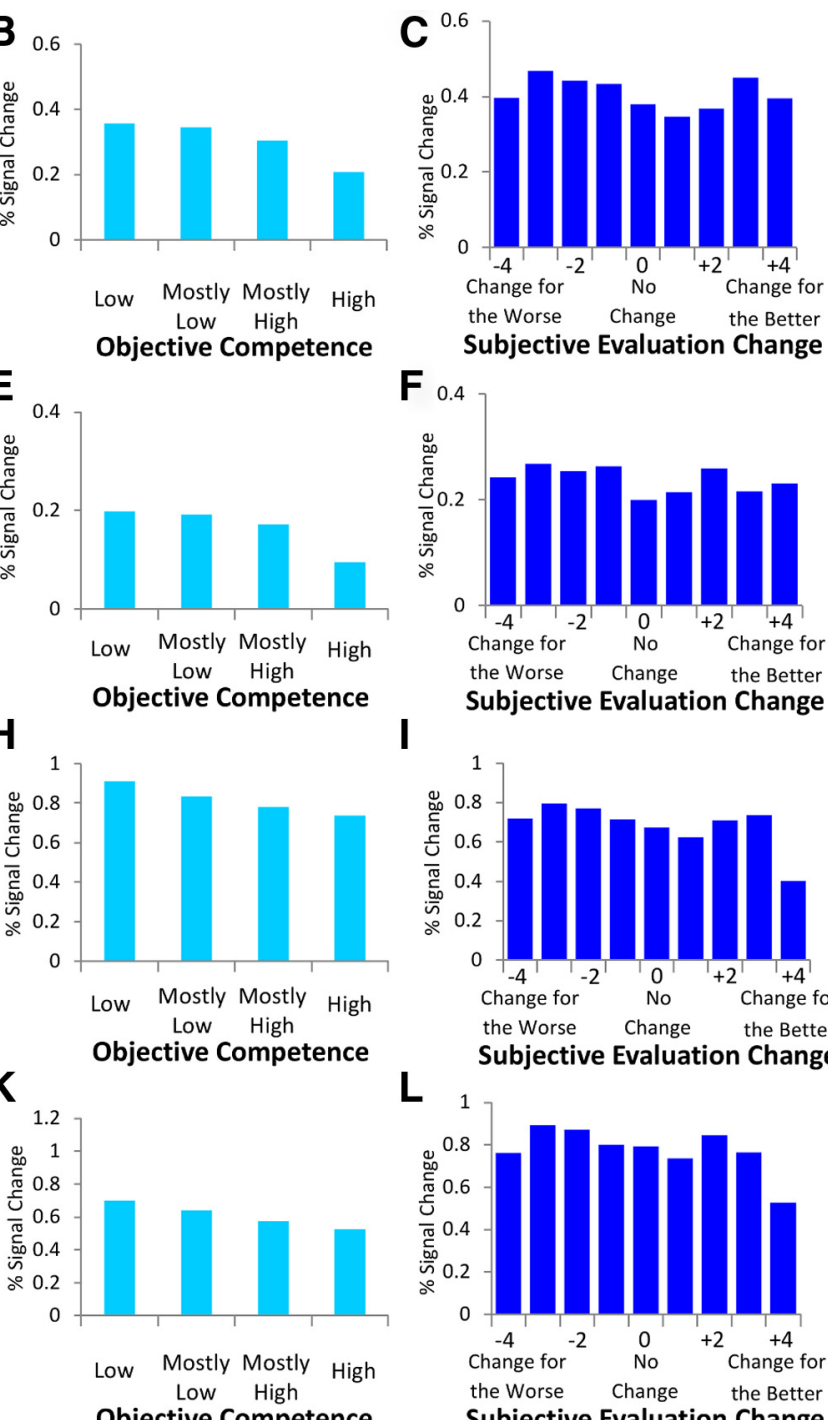

$\mathbf{F}_{0}$

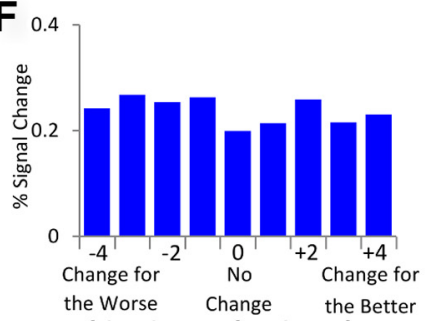

I

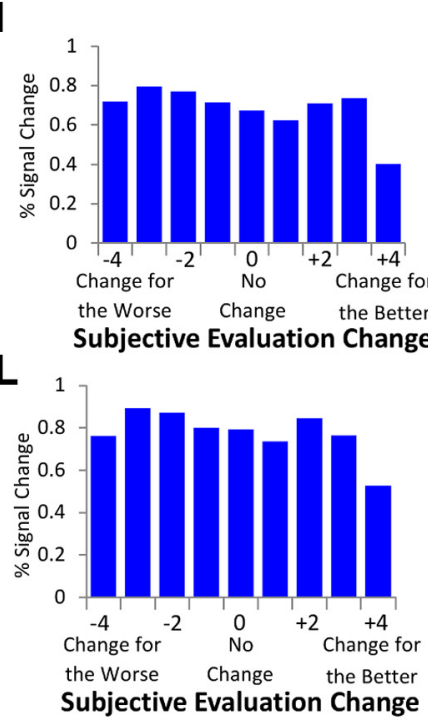

Figure 4. Illustration of parametric neural modulation and seeing a candidate for the worse. $A, D, G, J$, Depict voxels showing a parametric relation to experimentally manipulated competency-related information (light blue), changes in subjective competence evaluations (dark blue), or both effects (purple). $\boldsymbol{B}, \boldsymbol{E}, \boldsymbol{H}, \boldsymbol{K}$, Average percentage signal change is plotted in relation to experimentally manipulated levels of competence (averaged across light blue and purple voxels). $\boldsymbol{C}, \boldsymbol{F}, \boldsymbol{I}, \boldsymbol{L}$, Average percentage signal change is plotted in relation to how much participants had an unfavorable change of heart in their final evaluations of a candidate (averaged across dark blue and purple voxels). Negative values on the horizontal axis indicate that the final evaluation of the candidate's competence was lower than the initial evaluation. 
A

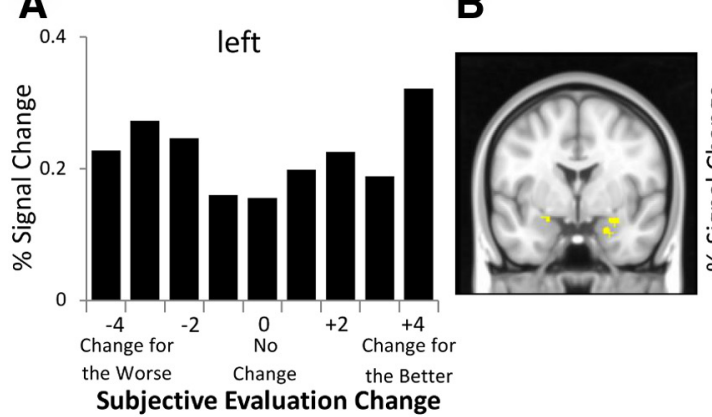

C

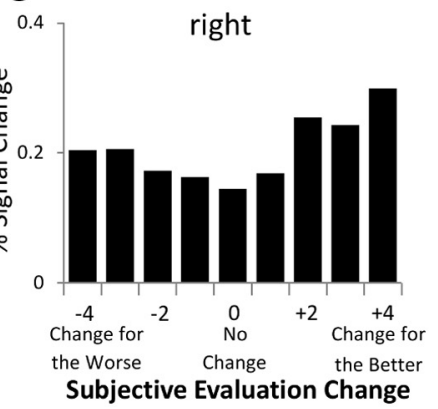

Figure 5. Illustration of quadratic amygdala modulation for evaluation change. Activation in left and right amygdala increases to the degree that evaluations change in either direction (for the better or for the worse). $\boldsymbol{A}, \boldsymbol{C}$, Average percentage signal change is plotted in relation to how much final evaluations rose or fell from the initial evaluation. Negative values indicate that the final evaluation of the candidate's competence was lower than the initial evaluation; positive values indicate the extent to which final evaluations of competence were higher than the initial evaluation. $\boldsymbol{B}$, Amygdala voxels showing a quadratic relation to changes in subjective competence evaluations.

change in any direction (amygdala), the findings show that changing your mind about someone is not simply a matter of estimating how much new information should change initial impressions. At the neural level, seeing a job candidate for the better was somewhat distinguishable from seeing a job candidate for the worse. New information about job candidates that led to an improvement in evaluation was processed using neural regions (e.g., right LPFC) that were distinct from those used to process new information that led to worsening impressions (e.g., MPFC and lateral temporal cortex). The behavioral data suggest that the roles of these regions are not necessarily in opposition to the role of left LOFC discussed above: participants incorporated new evidence and yet were still somewhat influenced by their initial impressions. The findings cast a different light on previous interpretations of how these neural regions support social evaluation.

The findings suggest a departure from the view that LPFC's role in person evaluation is to suppress pre-existing negative attitudes (e.g., race-based), a characterization that has arisen from investigating person evaluation as a one-time event (Richeson et al., 2003; Cunningham et al., 2004; Knutson et al., 2007). In the current study, LPFC's role did not require an initially negative impression. Instead, LPFC tracked the extent to which someone is seen for the better regardless of whether initial impressions were positive or negative. If LPFC is playing a similar role in the current study and previous research on race-based person evaluation, then it may reflect cognitive effort or complexity. In situations such as a job interview, people may discount positive statements made during the interview because there is a situational demand for candidates to say "stock" positive statements to portray themselves in a favorable light (Reeder and Brewer, 1979). Therefore, during trials where impressions changed for the better, participants may have corrected their tendency to discount the positive statements. In this way, positive job interview information that actually had an impact on final evaluations may have been processed using greater cognitive effort or complexity (Reeder and Brewer, 1979; Ybarra, 2002), just as suppressing a race-based attitude may require cognitive effort or complexity (Richeson et al., 2003). This interpretation is consistent with the association between LPFC activity and complex cognitive operations found in numerous domains (Christoff et al., 2001; Kroger et al., 2002; Bhanji et al., 2010). However, it might also be that the LPFC region associated with suppressing race-based attitudes may be different from the region found in the current study. To develop neural models of person evaluation, future research is needed to better understand if the LPFC relation to impression change is modulated by cognitive effort and whether LPFC acts within a similar or different neural network when suppressing the expression of pre-existing negative attitudes.

The current study also shows that neural regions known to respond to new or inconsistent information about a person do not necessarily predict any change in your evaluation of that person. MPFC, striatum, and lateral temporal cortex are associated with evaluating a number of person characteristics (Mitchell et al., 2005, 2006; Ochsner et al., 2005; Freeman et al., 2010; Baron et al., 2011) and respond to unexpected or inconsistent information about a person (Harris and Fiske, 2010; Baron et al., 2011; Cloutier et al., 2011; Ma et al., 2012; Mende-Siedlecki et al., 2013). This previous research has not specifically linked activity in these regions to social evaluations that change for the better or worse after an initial impression. In the current study, MPFC, striatum, and lateral temporal cortex activity responded to parametric manipulations of negative information (i.e., objective information about incompetence) and predicted the degree of subjective change in evaluation of job candidates so long as it was in an unfavorable direction. These findings illustrate the importance of studying person evaluation as it unfolds from first impression to subsequent evaluations as well as considering whether evaluations change for the better or the worse (Reeder and Brewer, 1979; Ybarra, 2002). Together, the current findings on LPFC, MPFC, striatum, and lateral temporal cortex suggest that a complete picture of the neural basis of social evaluation will require an understanding of how evaluators treat positive versus negative information in a differential manner.

Finally, the current investigation of dynamic person evaluation provides a novel extension of the amygdala's role in person evaluation. Although initially described as a marker of negative evaluations in humans, recent research has shown that amygdala activation shows a quadratic relation to the extremity of evaluation (i.e., both positive and negative; Cunningham et al., 2008; Said et al., 2009; Schiller et al., 2009). The current study finds a similar quadratic pattern of amygdala activation in relation to the extremity of evaluation changes for the better or for the worse. Together, the findings suggest that amygdala plays an important, but less specific, role in evaluation change than LPFC, MPFC, striatum, and lateral temporal cortex.

The current study investigated a dynamic of everyday social interaction: the (not always welcome) influence of a person's physical attractiveness on evaluations of their personality. The damage done by initial reactions to physical attractiveness is predicted by LOFC modulation. But impressions do not end at initial reactions; we may see someone for the better or the worse in light of new information. Favorable and unfavorable "changes of heart" are somewhat distinguishable at the neural level. The extremity of evaluation change (in any direction) quadratically modulates amygdala activation. Yet seeing someone for the better parametrically modulates LPFC activation, whereas seeing someone for the worse parametrically modulates MPFC, lateral temporal cortex, and striatal activation. The results illustrate how 
much there is to be learned when social evaluation is treated as much more than a one-time, static impression. Future research that gives the dynamics of social-evaluation their due is needed to more fully develop neural models of social evaluation.

\section{References}

Baron SG, Gobbini MI, Engell AD, Todorov A (2011) Amygdala and dorsomedial prefrontal cortex responses to appearance-based and behaviorbased person impressions. Soc Cogn Affect Neurosci 6:572-581. CrossRef Medline

Beer JS, Stallen M, Lombardo MV, Gonsalkorale K, Cunningham WA, Sherman JW (2008) The Quadruple Process model approach to examining the neural underpinnings of prejudice. Neuroimage 43:775-783. CrossRef Medline

Bhanji JP, Beer JS, Bunge SA (2010) Taking a gamble or playing by the rules: dissociable prefrontal systems implicated in probabilistic versus deterministic rule-based decisions. Neuroimage 49:1810-1819. CrossRef Medline

Büchel C, Holmes AP, Rees G, Friston KJ (1998) Characterizing stimulusresponse functions using nonlinear regressors in parametric fMRI experiments. Neuroimage 8:140-148. CrossRef Medline

Casey BJ, Trainor RJ, Orendi JL, Schubert AB, Nystrom LE, Giedd JN, Castellanos FX, Haxby JV, Noll DC, Cohen JD, Forman SD, Dahl RE, Rapoport JL (1997) A developmental functional MRI study of prefrontal activation during performance of a go-no-go task. J Cogn Neurosci 9:835-847. CrossRef

Christoff K, Prabhakaran V, Dorfman J, Zhao Z, Kroger JK, Holyoak KJ, Gabrieli JD (2001) Rostrolateral prefrontal cortex involvement in relational integration during reasoning. Neuroimage 14:1136-1149. CrossRef Medline

Cloutier J, Gabrieli JD, O'Young D, Ambady N (2011) An fMRI study of violations of social expectations: when people are not who we expect them to be. Neuroimage 57:583-588. CrossRef Medline

Croft KE, Duff MC, Kovach CK, Anderson SW, Adolphs R, Tranel D (2010) Detestable or marvelous? Neuroanatomical correlates of character judgments. Neuropsychologia 48:1789-1801. CrossRef Medline

Cunningham WA, Johnson MK, Raye CL, Chris Gatenby J, Gore JC, Banaji MR (2004) Separable neural components in the processing of black and white faces. Psychol Sci 15:806-813. CrossRef Medline

Cunningham WA, Van Bavel JJ, Johnsen IR (2008) Affective flexibility. Psychol Sci 19:152-160. CrossRef Medline

Desikan, RS, Ségonne F, Fischl B, Quinn BT, Dickerson BC, Blacker D, Buckner RL, Dale AM, Maguire RP, Hyman BT, Albert MS, Killiany RJ (2006) An automated labeling system for subdividing the human cerebral cortex on MRI scans into gyral based regions of interest. Neuroimage 31:968980. CrossRef Medline

Dion K, Berscheid E, Walster E (1972) What is beautiful is good. J Pers Soc Psychol 24:285-290. CrossRef Medline

Eagly AH, Ashmore RD, Makhijani MG, Longo LC (1991) What is beautiful is good, but ... A meta-analytic review of research on the physical attractiveness stereotype. Psychol Bull 110:109. CrossRef

Elliott R, Dolan RJ, Frith CD (2000) Dissociable functions in the medial and lateral orbitofrontal cortex: evidence from human neuroimaging studies. Cereb Cortex 10:308-317. CrossRef Medline

Feingold A (1992) Good-looking people are not what we think. Psychol Bull 111:304-341. CrossRef

Freeman JB, Schiller D, Rule NO, Ambady N (2010) The neural origins of superficial and individuated judgments about ingroup and outgroup members. Hum Brain Mapp 31:150-159. Medline

Harris LT, Fiske ST (2010) Neural regions that underlie reinforcement learning are also active for social expectancy violations. Soc Neurosci 5:76-91. CrossRef Medline

Jackson LA, Hunter JE, Hodge CN (1995) Physical attractiveness and intellectual competence: a meta-analytic review. Soc Psychol Q 58:108-122. CrossRef

Kim H, Choi MJ, Jang IJ (2012) Lateral OFC activity predicts decision bias due to first impressions during ultimatum games. J Cogn Neurosci 24: 428-439. CrossRef Medline

Knutson KM, Mah L, Manly CF, Grafman J (2007) Neural correlates of automatic beliefs about gender and race. Hum Brain Mapp 28:915-930. CrossRef Medline

Kriegeskorte N, Simmons WK, Bellgowan PS, Baker CI (2009) Circular analysis in systems neuroscience: the dangers of double-dipping. Nat Neurosci 12:535-540. CrossRef Medline

Kroger JK, Sabb FW, Fales CL, Bookheimer SY, Cohen MS, Holyoak KJ (2002) Recruitment of anterior dorsolateral prefrontal cortex in human reasoning: a parametric study of relational complexity. Cereb Cortex 12 : 477-485. CrossRef Medline

Ma N, Vandekerckhove M, Baetens K, Van Overwalle F, Seurinck R, Fias W (2012) Inconsistencies in spontaneous and intentional trait inferences. Soc Cogn Affect Neurosci 7:937-950. CrossRef Medline

Mende-Siedlecki P, Cai Y, Todorov A (2013) The neural dynamics of updating person impressions. Soc Cogn Affect Neurosci, in press.

Mitchell JP, Neil Macrae C, Banaji MR (2005) Forming impressions of people versus inanimate objects: social-cognitive processing in the medial prefrontal cortex. Neuroimage 26:251-257. CrossRef Medline

Mitchell JP, Cloutier J, Banaji MR, Macrae CN (2006) Medial prefrontal dissociations during processing of trait diagnostic and nondiagnostic person information. Soc Cogn Affect Neurosci 1:49-55. CrossRef Medline

Nichols T, Brett M, Andersson J, Wager T, Poline JB (2005) Valid conjunction inference with the minimum statistic. Neuroimage 25:653-660. CrossRef Medline

Ochsner KN, Beer JS, Robertson ER, Cooper JC, Gabrieli JD, Kihsltrom JF, D'Esposito M (2005) The neural correlates of direct and reflected selfknowledge. Neuroimage 28:797-814. CrossRef Medline

O’Doherty J, Winston J, Critchley H, Perrett D, Burt DM, Dolan RJ (2003) Beauty in a smile: the role of medial orbitofrontal cortex in facial attractiveness. Neuropsychologia 41:147-155. CrossRef Medline

Reeder GD, Brewer MB (1979) A schematic model of dispositional attribution in interpersonal perception. Psychol Rev 86:61-79. CrossRef

Richeson JA, Baird AA, Gordon HL, Heatherton TF, Wyland CL, Trawalter S, Shelton JN (2003) An fMRI investigation of the impact of interracial contact on executive function. Nat Neurosci 6:1323-1328. CrossRef Medline

Said CP, Baron SG, Todorov A (2009) Nonlinear amygdala response to face trustworthiness: contributions of high and low spatial frequency information. J Cogn Neurosci 21:519-528. CrossRef Medline

Schiller D, Freeman JB, Mitchell JP, Uleman JS, Phelps EA (2009) A neural mechanism of first impressions. Nat Neurosci 12:508-514. CrossRef Medline

Smith SM, Jenkinson M, Woolrich MW, Beckmann CF, Behrens TEJ, Johansen-Berg H, Bannister PR, De Luca M, Drobnjak I, Flitney DE, Niazy RK, Saunders J, Vickers J, Zhang Y, De Stefano N, Brady JM, Matthews PM (2004) Advances in functional and structural MR image analysis and implementation as FSL. Neuroimage 23:S208-S219. CrossRef Medline

Srivastava S, Guglielmo S, Beer JS (2010) Perceiving others' personalities: examining the dimensionality, assumed similarity to the self, and stability of perceiver effects. J Pers Soc Psychol 98:520-534. CrossRef Medline

Ybarra O (2002) Naive causal understanding of valenced behaviors and its implications for social information processing. Psychol Bull 128:421441. CrossRef Medline 